\title{
O-GlcNAc modification is associated with insulin sensitivity in the whole blood of healthy young adult males
}

\author{
Jason P Myslicki ${ }^{1}$, Jane Shearer ${ }^{1,2}$, Dustin S Hittel ${ }^{1}$, Curtis C Hughey ${ }^{2}$ and Darrell D Belke ${ }^{1 *}$
}

\begin{abstract}
Background: Hemoglobin A1c (HbA1c) is the predominant diagnostic tool for diabetes diagnosis and progression. However, it has proven to be insensitive at pre-diabetic threshold values. O-linked- $\beta$ - $N$-acetylglucosamine (O-GlcNAc) modification has emerged as a sensitive biomarker. The purpose of this study was to explore the sensitivity of O-GlcNAc expression as a potential marker of early metabolic dysfunction in a young adult population. Healthy, young males (18-35 y) from the Assessing Inherited Metabolic syndrome Markers in the Young study (AIMMY), were divided into low $(\mathrm{LH}, 0.60)$ or high $(\mathrm{HH}, 1.61)$ homeostatic model assessment of insulin resistance (HOMA-IR) cohorts.

Findings: The relationships between a panel of anthropometric, metabolic measures and whole blood global protein O-GIcNAc was examined. O-GIcNAc and O-GlcNAc transferase (OGT) levels were quantified by immunoblotting and compared to anthropometric measures: body mass index (BMI), percentage body fat, aerobic fitness, blood glucose, triglycerides, HDL, insulin, and HbA1c. HOMA-IR cohorts showed no differences in BMl, blood glucose or HbA1c, but differed in percent body fat, plasma triglycerides, and circulating insulin. Greater O-GlcNAc expression was observed in the whole blood of HH compared to LH. Moreover, a positive association between HOMA-IR and O-GIcNAc emerged, while no relationship was found between $\mathrm{HbA1c}$ and HOMA-IR. This effect was not related to OGT expression.
\end{abstract}

Conclusions: Results indicate that O-GlcNAc has a greater sensitivity to metabolic status compared to HbA1c in this population. O-GlcNAc has the potential to serve as a screening tool for predicting future metabolic disturbances in a young healthy adult population free of any clinically relevant pathologies.

Keywords: Young adult, Metabolism, HOMA-IR, Obesity, Insulin resistance

\section{Introduction}

Current estimates indicate there are 347 million diagnosed cases of diabetes worldwide, $90 \%$ of these being attributable to type 2 diabetes (T2D) [1]. Research establishes that metabolic diseases, including T2D are often initiated in childhood. In fact, the extent of metabolic disease risk in children and young adults is correlated with the presence of the same risk factors identified in adults including obesity and insulin resistance [2].

At present, haemoglobin A1c (HbA1c) is the preferred biomarker for diabetes diagnosis and monitoring; however, this marker has proven to be insensitive at pre-diabetic and diabetic threshold values [3]. As a result, there has

\footnotetext{
* Correspondence: dbelke@ucalgary.ca

${ }^{1}$ Faculty of Kinesiology, University of Calgary, 3300 University Drive NW, Calgary, Alberta T2N 4N1, Canada

Full list of author information is available at the end of the article
}

been an increased prevalence of undiagnosed diabetes, thereby increasing the potential for complications. Recently, the post-translational protein modification, Olinked $\beta$ - $N$-acetylglucosamine (O-GlcNAc), has emerged as a potential biomarker for T2D. Protein O-GlcNAc is the post-translational modification of intracellular proteins by the addition or removal of O-GlcNAc mediated by $\beta$-N-acetylglucosaminyltransferase (OGT) and $\beta$-Nacetylglucosaminidase (OGA) in a manner analogous to phosphorylation [4]. As the O-GlcNAc pathway utilizes uridine diphosphate $\mathrm{N}$-acetylglucosamine from the hexosamine biosynthetic pathway, its study has been closely linked to circulating glucose levels and diabetes, where it is thought to play a role in mediating insulin resistance [5,6].

Aberrant protein O-GlcNAc modification has been associated with hyperglycemia, insulin resistance and 
metabolic dysfunction $[4,7]$. To date, studies have examined changes in the O-GlcNAc of erythrocytes and leukocytes of healthy, pre-diabetic and overtly diabetic adults in order to gain an enhanced understanding of this modification as it relates to the development of the disease $[4,7,8]$. These studies show that in overt diabetes, O-GlcNAc analysis has the potential to identify metabolic disturbances better than HbA1c $[7,8]$. However, nothing is known about O-GlcNAc levels in a clinically healthy young, asympotmatic adult population. Study aims were to explore the relationships between a panel of anthropometric and metabolic parameters with whole blood O-GlcNAc, OGT and OGA in a sample of healthy young adults.

\section{Methods}

Whole blood samples $(n=24)$ were collected from the Assessing Inherited Markers of Metabolic Syndrome in the Young (AIMMY) cohort at the University of Calgary and approved by the Conjoint Health Research Ethics Board (ID: E23521). Individual subjects were chosen from the entire AIMMY cohort $(n=122)$ based on their Homeostatic Model of Assessment - Insulin Resistance (HOMA-IR) classification, being in either below lowest (LL, P25, <0.845) or greatest ( $\mathrm{HH}, \mathrm{P} 75,>1.61)$ quartiles for this measure. HOMA-IR calculated as: [HOMA-IR = [Fasting glucose $\left(\mathrm{mmol} \cdot \mathrm{L}^{-1}\right) \times$ fasting insulin $\left.(\mathrm{pmol})\right] /$ 22.5]. The inclusion criteria were 1) age 18-35 years, 2) post-pubescent and 3) willing and able to provide informed consent. All subjects were considered healthy as previously outlined [9]. The study required subjects to fast for $12 \mathrm{~h}$ prior to blood collection [9]. Body mass index (BMI), percentage body fat (\%), blood measures including glucose, triglycerides, high density lipoprotein cholesterol, insulin, and HbA1c were collected and analyzed as described [9].

Whole blood samples were collected and immediately frozen. In preparation for immunoblotting, blood samples were homogenized in a lysis buffer ( $\mathrm{pH}$ 7.4) consisting of $20 \mathrm{mM} \mathrm{NaCl}, 20 \mathrm{mM}$ Tris- $\mathrm{HCl}, 0.1 \mathrm{mM}$ EDTA, $1 \%$ Triton X-100, 0.5\% (wt./vol.) sodium deoxycholate, and $0.1 \% \beta$-mercaptoethanol (vol./vol.) in the presence of phosphatase inhibitor, protease inhibitor, and PUGNAc (N-acetylhexosaminidase inhibitor, Sigma-Aldrich, St. Louis, MO, A7229) to inhibit O-GlcNAcase activity. Blood proteins were detected as previously described $[10,11]$. Primary antibodies included anti-RL2 (1:1000; Abcam, ab2739), anti-OGT (1:1000; DM-17 SigmaAldrich, St. Louis, MO), and anti-OGA (1:1000; Santa Cruz Biotech, sc-135093) with glyceraldehyde 3-phosphate dehydrogenase (anti-GAPDH) (1:1000; Abcam, ab9485) serving as a control. Secondary antibodies were as follows: anti-RL2: goat anti-mouse IgG HRP conj. (1:2000, Thermo Scientific, 32430); and anti-OGT, anti-OGA, and anti-GAPDH: goat anti-rabbit IgG HRP (1:2000, Cell Signaling Technology, 7074). Signals were detected employing chemiluminesce (West Femto, Thermo Fisher Scientific) and then exposed and analyzed using Syngene ChemiGenius ${ }^{2}$ Bio Imaging System.

Subject characteristics and metabolic markers (Table 1, Figure 1B) were compared employing independent $t$ tests at a $\alpha=0.05$ significance level (SPSS Statistics V20). The Pearson Product-moment Correlation Coefficient was determined and compared to a critical value for a two-tailed test (Figures 1C-E) [12]. All data are reported as mean \pm standard error of measurement (SEM).

\section{Results}

Subject characteristics are presented in Table 1. HOMAIR for the $\mathrm{LH}$ and $\mathrm{HH}$ group were $0.5 \pm 0.1$ and $1.9 \pm 0.2$ respectively. These values were much lower than the widely adopted HOMA-IR cut-off of 2.60 for insulin resistance [13]. No individuals were obese (BMI $>30)$ or prediabetic. The current diabetes diagnostic method, HbA1c, was unable to distinguish the two groups, $\mathrm{LH}$ and $\mathrm{HH}$, with respect to insulin resistance ( $\mathrm{p}>0.05)$.

Global O-GlcNAc modification was greater in $\mathrm{HH}$ compared to LH $(\mathrm{p}=0.02)$ (Figure 1A, B). Results show that this effect was not related to a difference in OGT expression (Figure 1A) and we were unable to attain any consistent OGA signal with immunoblotting, hence we did not include these data. OGA has been shown to be vulnerable to freeze thaw cycles $[7,14]$, possibly explaining why no signal was detected. All correlations are exhibited in Figure $1 \mathrm{C}$ including the noteworthy positive correlation between O-GlcNAc and HOMA-IR $(r=0.68$, $\mathrm{p}<0.05$ ) (Figure 1C). Traditional metabolic disease

Table 1 Baseline characteristics and metabolic markers of all subjects and stratified by low and high homeostatic model assessment, insulin resistance (HOMA-IR) scores

\begin{tabular}{|c|c|c|c|}
\hline & $\begin{array}{l}\text { All Subjects } \\
(n=24)\end{array}$ & $\begin{array}{l}\text { Low HOMA-IR } \\
(n=12)\end{array}$ & $\begin{array}{l}\text { High HOMA-IR } \\
(n=12)\end{array}$ \\
\hline Age (years) & $24 \pm 1$ & $24 \pm 1$ & $24 \pm 1$ \\
\hline Mean HOMA-IR & $1.2 \pm 0.2$ & $0.5 \pm 0.1$ & $1.9 \pm 0.2$ \\
\hline $\mathrm{BMI}$ & $24.7 \pm 0.6$ & $23.9 \pm 0.8$ & $25.5 \pm 0.7$ \\
\hline Body Fat (\%) & $16.3 \pm 1.4$ & $12.2 \pm 0.9$ & $20.3 \pm 2.2^{*}$ \\
\hline Glucose (mM) & $4.5 \pm 0.1$ & $4.6 \pm 0.1$ & $4.5 \pm 0.1$ \\
\hline Triglycerides (mM) & $1.0 \pm 0.1$ & $0.8 \pm 0.1$ & $1.2 \pm 0.1^{*}$ \\
\hline HDL Cholesterol (mM) & $1.5 \pm 0.1$ & $1.7 \pm 0.1$ & $1.4 \pm 0.1$ \\
\hline Insulin (pM) & $43.4 \pm 6.0$ & $19.8 \pm 2.3$ & $65.1 \pm 6.6^{*}$ \\
\hline $\mathrm{HbA1c}(\%)$ & $5.5 \pm 0.1$ & $5.5+0.1$ & $5.5+0.1$ \\
\hline $\begin{array}{l}\mathrm{VO}_{2 \text { peak }}(20 \mathrm{~s}) \\
\left(\mathrm{ml} \cdot \mathrm{kg}^{-1} \cdot \mathrm{min}^{-1}\right)\end{array}$ & $49.1 \pm 1.8$ & $52.2 \pm 2.4$ & $45.7 \pm 2.5$ \\
\hline
\end{tabular}

Abbreviations are as follows: body mass index $\left(\mathrm{BMl}, \mathrm{kg} \cdot \mathrm{m}^{-2}\right)$, high-density lipoprotein cholesterol (HDL, mM), glycated hemoglobin (HbA1c, \%), aerobic capacity $\left(\mathrm{VO}_{2}\right.$ peak, $\left.\mathrm{ml} \cdot \mathrm{kg}^{-1} \cdot \mathrm{min}^{-1}\right)$. Values are means $\pm \mathrm{SEM}$. ${ }^{*}$ Indicates there is a significant difference $(p<0.05)$ between low and high HOMA-IR groups. 

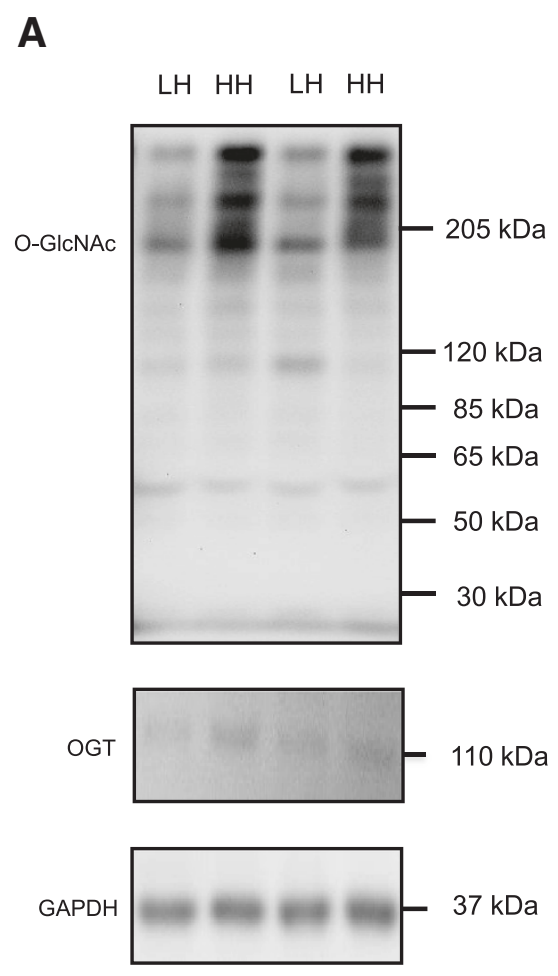

B

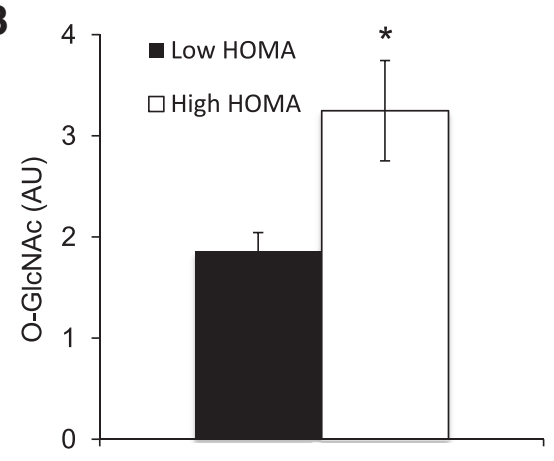

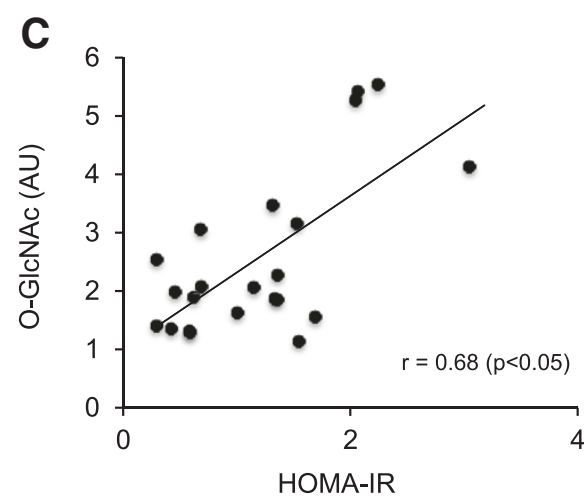
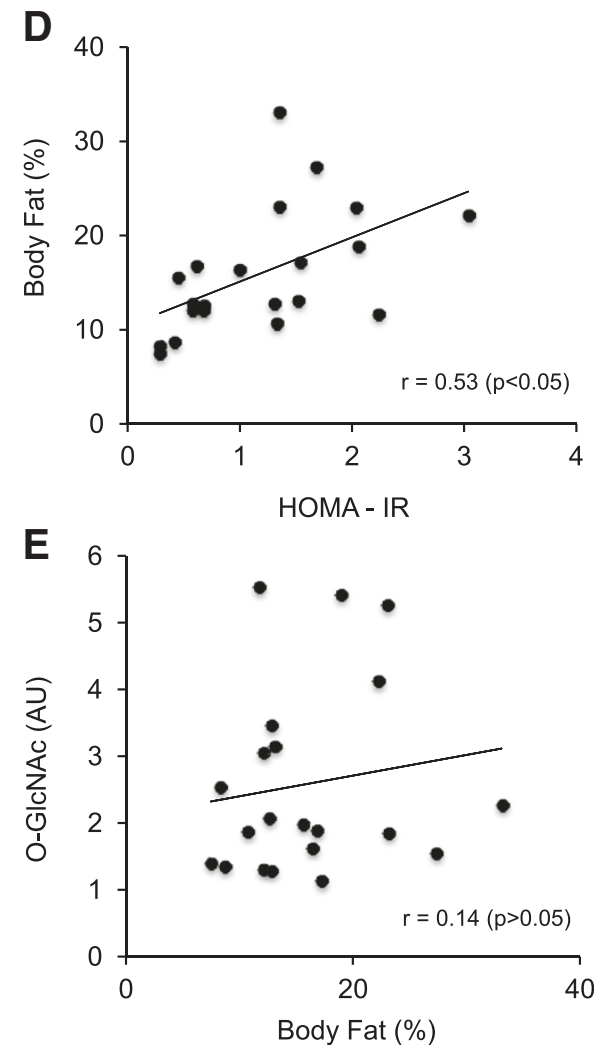

Figure 1 Analysis of Blood for O-GIcNAc levels in Relation to Anthropometric Measurements. A) Analysis of whole blood comparing subjects with low $(\mathrm{LH})$ and high $(\mathrm{HH})$ homeostatic model assessment for insulin resistance (HOMA-IR) for global O-GlcNAcylation (O-GlcNAc), $\beta$-N-acetylglucosaminyltransferase (OGT), and glyceraldehyde 3-phosphate dehydrogenase (GAPDH) in arbitrary units (AU). B) Quantification of global O-GICNAc for LH and HH. C) Linear regression analysis of O-GlcNAc and HOMA-IR. D) Linear regression analysis of body fat percentage (\%) and HOMA-IR. E) Linear regression analysis of O-GICNAc and body fat percentage (\%). All data represents $n=24$. ${ }^{*}$ Indicates $p<0.05$.

markers, such as BMI, body fat percentage, plasma HDL and triglycerides all had moderate to strong relationships with HOMA-IR. The relationship between body fat percentage and HOMA-IR is shown in Figure 1D $(r=0.53$, $\mathrm{p}<0.05)$. The relationship between O-GlcNAc and body fat percentage is shown in Figure $1 \mathrm{E}(\mathrm{r}=0.14, \mathrm{p}>0.05)$. There was no statistically significant difference in $\mathrm{VO}_{2}$ peak (20s) between $\mathrm{LH}$ and $\mathrm{HH}(\mathrm{p}=0.07)$, nor a relationship between O-GlcNAc and $\mathrm{VO}_{2}$ (Additional file 1: Table S1).

\section{Discussion and conclusion}

Many metabolic disease risk factors, including insulin resistance begin to accumulate in young adulthood [15]. Consequently, there is an acute need to establish a sensitive biomarker of diabetes prior to initiation of the disease in this population. This study explored the relationship between whole protein O-GlcNAc and HOMA-IR in a healthy young adult population, free of any clinically relevant pathology involving metabolism. O-GlcNAc may be an ideal marker because intracellular O-GlcNAc 
modification of proteins serves as a cellular tool for nutrient sensing [16] and cellular protection [17]. In metabolic disease states such as type 2 diabetes, hyperglycemia drives O-GlcNAc and the chronic modification of selected proteins including those involved in insulin signalling [18]. The chronic modification of certain proteins has also been linked to T2D aetiology and progression $[4,5,16]$.

Results of the present study show that O-GlcNAc modification can be used to discriminate between $\mathrm{LH}$ and $\mathrm{HH}$ in a young, healthy population. In contrast, no differences were observed in HbA1c, the current diabetes diagnostic gold standard. This suggests that that $\mathrm{O}$ GlcNAc analysis exhibits greater sensitivity and could potentially serve as a better screening tool for identifying the risk for future metabolic disturbances in a nonovertly diabetic population. These results concur with those of Springhorn et al. [8] who demonstrated that leukocytes from both pre-diabetic and diabetic individuals have elevated global O-GlcNAc compared to healthy controls. The positive correlation between $\mathrm{O}$ GlcNAc and HOMA-IR suggests that the two indices will continue to rise simultaneously but, due to unique personal baseline measurements, determining a threshold cut-off for identifying a given pathology is unrealistic. As such, O-GlcNAc modification may be better utilized as a screening tool and for individual monitoring.

The lack of difference in OGT expression suggests that changes in O-GlcNAc modification are likely due to other enzymatic activity, such as decreased OGA in the $\mathrm{HH}$ group, or simply elevated circulating glucose levels and subsequently increased flux through the hexosamine biosynthetic pathway, which is associated with insulin resistance. Biochemically, altered glucose metabolism is a requisite for aberrant O-GlcNAc expression and, in general, nutritional intake, whether it is fatty acid, nucleotide, or glucose, has demonstrated an effect on O-GlcNAcylation as UDPGlcNAc, the donor molecular for O-GlcNAc, sits at the nexus of these metabolic pathways [19]. As such, lifestyle factors such as diet and exercise should be expected to play a key role in O-GlcNAc modification. In the subjects examined, O-GlcNAc modification did not correlate with $\mathrm{VO}_{2}$ max suggesting that O-GlcNAc modification does not provide insight into an individual's aerobic capacity or pulmonary functional competence. To date, relatively little is known regarding the effect of chronic exercise on O-GlcNAc modification in blood to help explain this relationship.

Given the lack of studies examining differences in protein O-GlcNAc in a young clinically healthy population, especially with respect to susceptibility towards chronic disease development later in life, our observations provide a foundation for long-term studies of O-GlcNAc as a sensitive screening tool for risk of future metabolic disturbances.
We acknowledge the practical limitations associated with administering protein O-GlcNAc quantification via immunoblot analysis to large populations, nonetheless this preliminary research demonstrates the utility of O-GlcNAc analysis as a screening tool. Moving forward, prospective studies following O-GlcNAc modifications over time along with changes in health status, insulin resistance, or the progression of diagnosed diabetes are warranted.

\section{Additional file}

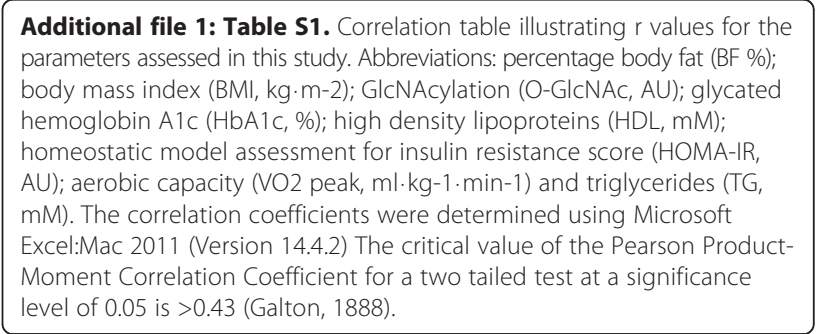

Competing interests

The authors declare that they have no competing interests.

\section{Authors' contributions}

JPM, DDB and CCH performed the experiments and collected the data. JM is the primary author. JS, DB and DSH assisted with manuscript writing, statistical analysis and data interpretation. All authors read and approved the final manuscript.

\section{Acknowledgements}

The authors gratefully acknowledge the financial support of the National Science and Engineering Research Council of Canada (JS, DSH). JS holds salary support awards from Alberta Innovates Health Solutions. The authors thank Virginia L. Johnsen for her outstanding technical support.

\section{Author details}

${ }^{1}$ Faculty of Kinesiology, University of Calgary, 3300 University Drive NW, Calgary, Alberta T2N 4N1, Canada. ${ }^{2}$ Department of Biochemistry and Molecular Biology, Faculty of Medicine, University of Calgary, Calgary, Alberta T2N 1N4, Canada.

Received: 10 April 2014 Accepted: 27 August 2014

Published: 9 September 2014

\section{References}

1. Danaei G, Finucane MM, Lu Y, Singh GM, Cowan MJ, Paciorek CJ, Lin JK, Farzadfar F, Khang YH, Stevens GA, Rao M, Ali MK, Riley LM, Robinson CA, Ezzati M: National, regional, and global trends in fasting plasma glucose and diabetes prevalence since 1980: systematic analysis of health examination surveys and epidemiological studies with 370 country-years and 2.7 million participants. Lancet 2011, 378:31-40.

2. Berenson GS, Srinivasan SR, Bao W, 3rd Newman WP, Tracy RE, Wattigney WA: Association between multiple cardiovascular risk factors and atherosclerosis in children and young adults. The Bogalusa heart study. N Engl J Med 1998, 338:1650-1656.

3. Carson AP, Reynolds K, Fonseca VA, Muntner P: Comparison of A1C and fasting glucose criteria to diagnose diabetes among U.S. adults. Diabetes Care 2010, 33:95-97.

4. Wang Z, Park K, Comer F, Hsieh-Wilson LC, Saudek CD, Hart GW: Site-specific GlcNAcylation of human erythrocyte proteins: potential biomarker(s) for diabetes. Diabetes 2009, 58:309-317.

5. Issad T, Masson E, Pagesy P: O-GlcNAc modification, insulin signaling and diabetic complications. Diabetes Metab 2010, 36:423-435.

6. Vosseller K, Wells L, Lane MD, Hart GW: Elevated nucleocytoplasmic glycosylation by O-GlcNAc results in insulin resistance associated with 
defects in Akt activation in 3 T3-L1 adipocytes. Proc Natl Acad Sci U S A 2002, 99:5313-5318.

7. Park K, Saudek CD, Hart GW: Increased expression of beta-Nacetylglucosaminidase in erythrocytes from individuals with pre-diabetes and diabetes. Diabetes 2010, 59:1845-1850.

8. Springhorn C, Matsha TE, Erasmus RT, Essop MF: Exploring leukocyte O-GICNAcylation as a novel diagnostic tool for the earlier detection of type 2 diabetes mellitus. J Clin Endocrinol Metab 2012, 97:4640-4649.

9. Karlos A, Shearer J, Gnatiuk E, Onyewu C, Many G, Hoffman EP, Hittel DS: Effect of the SORT1 low-density lipoprotein cholesterol locus is sexspecific in a fit, Canadian young-adult population. Appl Physiol Nutr Metab 2013, 38:188-193.

10. Bennett CE, Johnsen VL, Shearer J, Belke DD: Exercise training mitigates aberrant cardiac protein O-GIcNAcylation in streptozotocin-induced diabetic mice. Life Sci 2013, 92:657-663.

11. Johnsen VL, Belke DD, Hughey CC, Hittel DS, Hepple RT, Koch LG, Britton SL, Shearer J: Enhanced cardiac protein glycosylation (O-GlcNAc) of selected mitochondrial proteins in rats artificially selected for low running capacity. Physiol Genomics 2013, 45:17-25.

12. Galton F: Co-relations and their measurement, chielfy from anthropometric data. Proc Roy Soc London 1888, 45:135-145.

13. Ascaso JF, Pardo S, Real JT, Lorente RI, Priego A, Carmena R: Diagnosing insulin resistance by simple quantitative methods in subjects with normal glucose metabolism. Diabetes Care 2003, 26:3320-3325.

14. Massaccesi L, Lombardo A, Venerando B, Tettamanti G, Goi G: Isoenzyme pattern and partial characterization of hexosaminidases in the membrane and cytosol of human erythrocytes. Clin Biochem 2007, 40:467-477.

15. Stehouwer $C D$, Henry RM, Ferreira I: Arterial stiffness in diabetes and the metabolic syndrome: a pathway to cardiovascular disease. Diabetologia 2008, 51:527-539.

16. Slawson C, Copeland RJ, Hart GW: O-GICNAC signaling: a metabolic link between diabetes and cancer? Trends Biochem Sci 2010, 35:547-555.

17. Wang ZV, Deng Y, Gao N, Pedrozo Z, Li DL, Morales CR, Criollo A, Luo X, Tan W, Jiang N, Lehrman MA, Rothermel BA, Lee AH, Lavandero S, Mammen PP, Ferdous A, Gillette TG, Scherer PE, Hill JA: Spliced X-box binding protein 1 couples the unfolded protein response to hexosamine biosynthetic pathway. Cell 2014, 156:1179-1192.

18. Whelan SA, Dias WB, Thiruneelakantapillai L, Lane MD, Hart GW: Regulation of insulin receptor substrate 1 (IRS-1)/AKT kinase-mediated insulin signaling by O-Linked beta-N-acetylglucosamine in $3 \mathrm{~T} 3-\mathrm{L} 1$ adipocytes. J Biol Chem 2010, 285:5204-5211.

19. Wells $L$, Vosseller $\mathrm{K}$, Hart GW: A role for $\mathrm{N}$-acetylglucosamine as a nutrient sensor and mediator of insulin resistance. Cell Mol Life Sci 2003, 60:222-228

doi:10.1186/1758-5996-6-96

Cite this article as: Myslicki et al: O-GlcNAc modification is associated with insulin sensitivity in the whole blood of healthy young adult males. Diabetology \& Metabolic Syndrome 2014 6:96.

\section{Submit your next manuscript to BioMed Central and take full advantage of:}

- Convenient online submission

- Thorough peer review

- No space constraints or color figure charges

- Immediate publication on acceptance

- Inclusion in PubMed, CAS, Scopus and Google Scholar

- Research which is freely available for redistribution

Submit your manuscript at www.biomedcentral.com/submit
C Biomed Central 